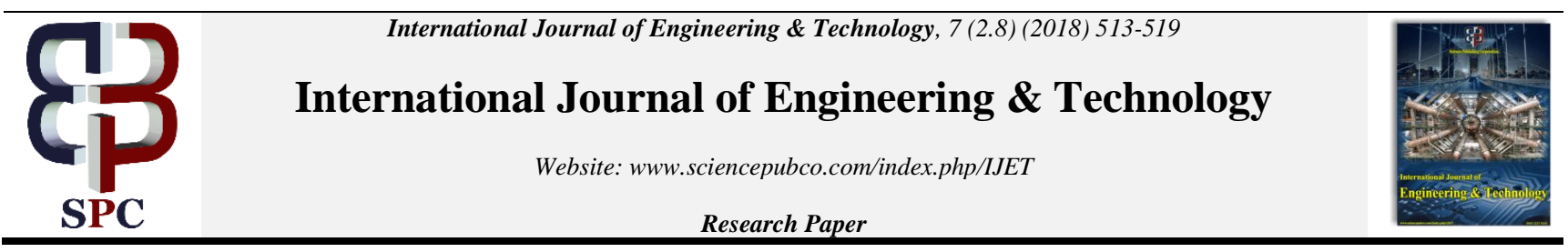

\title{
Optimal control of microgrid by decentralized controller
}

\author{
A. Subhashree ${ }^{*}$, K. Thenmalar $^{2},{ }^{3}$ K. Deepa ${ }^{3}$ \\ ${ }^{1,3} P G$ Scholar/Dept of EEE, Tiruchengode \\ ${ }^{2}$ Associate Professor/Dept of EEE, Tiruchengode \\ *Corresponding Author Email: asubhashree95@gmail.com
}

\begin{abstract}
The Microgrid is modern small range of centralized electricity system. It achieves specific local goal such as reliability, carbon emission reduction, diversification of energy sources, and cost reduction. Microgrids are an ideal way to integrate renewable sources. The Microgrid is motivated by many factors such that, the need to integrate more renewable power to mitigate the global warming., increasing interest in connecting more Distribution Generation as a way to postpone large investment in transmission and bulk generation and the need to increase the reliability of the power system overall to minimize distribution losses. In microgrid the power generation is mainly depends on renewable energy sources. The renewable sources are unpredictable, so to maintain the system stability in uncertain environment is very difficult. During the changes in microgrids, it is to satisfy the demand with minimum tracking time and to stabilize the system. In order minimize the tracking time for attaining the system stability, robust PID control method is proposed. The simulation has been done in MATLAB/Simulink with required formulation.
\end{abstract}

Keywords: Optimization Of Microgrid; DER-Distribution Energy Resource; MG-Maingrid;VSI-Multilevel Inverter.

\section{Introduction}

Modern world depends on a secure supply of energy. Growing concerns for primary energy availability. Aged infrastructure of current electrical transmission and distribution networks are increasingly challenging one. Because of entire system is controlled by the common grid control system. It is very difficult operation and very significant amounts of investment will be required to develop. At present the non-renewable energy sources is also very costly, so the renewable energy source integration is most important for future energy generation. The renewable energy generation is seasonal one, so it operation, control and storage is very critical operation. Instead of this problem the entire grid is splited in micro grid and the control is decentralized.

In future the electricity grids have to manage with changes in technology, in the values of society, in the environment and in economy. Thus, system security, operation safety, environmental protection, power quality, cost of supply and energy efficacy need to be inspected in new ways in response to changing requirements in a liberalized market environment [12]. The technology prove reliability, sustainability and cost effectiveness. The notion of smart grids refers to the evaluation of electricity grids. It is worth noting that power systems have always been "smart", especially at the transmission level [4]. The distribution level, however, is now experiencing an evaluation that needs more "smartness". In order to facilitate access to distributed generation on a high share, based on renewable energy source, either self-dispatched or dispatched by local distribution system operators enable local energy demand management, interacting with end-users through smart metering system benefits from technologies already applied in transmission grids, such as dynamic control techniques, so as to offer a higher overall level of power security, quality and reliability[15].

The distribution grids are being transformed from passive networks. In the sense that decision-making and control are distributed, and power flow is bidirectional. This type of network eases the integration of DGs, RES, demand side integration and energy storages technologies[14]. This creates opportunities for novel types of equipment and services. All of which would need to confirm to common protocols and standards. The main function of an active distribution network is to efficiency link power generation with consumer demands, allowing both to decide how best to operate in real-time. Power flow assessment, voltage control, protection require cost-competitive technologies, new communication systems with information and communication and communication technology playing a key role.

The decentralized controller is executing the proper algorithm for continuous operation and system voltage stability. The robust optimization is used to find tracking time for stability and number of iteration [6].

\section{Decentralized Topology}

\section{A. Robust Optimization}

The common grid is split into microgrids, and the power is generated from DGs. If the RES are more oscillated sources and seasonal one, so the battery storage and MG connection is more reliable operation of Microgrid. If the renewable energy is not present, then the load gets the supply from the MG side or if the microgrid produces the more power then to supply to the MG. The MG is always operating at conventional sources. The microgrid operates based on decentralized controller algorithm flow. The controller is operating the robust optimization. A proportionalintegral-derivative controller is a control loop feed back

mechanism widely used in industrial control system. A PID controller calculates an error value that is the different of measured value and set value. To minimize the error the controller adjusting the process through use of a manipulated variable. Three separate constant parameters are $\mathrm{P}, \mathrm{I}$ and $\mathrm{D}$, that is called proportional, integral and derivative respectively. These values can be interpreted in terms of time $\mathrm{P}$ depends on the present error, I on 
the accumulation of past errors, and D is a prediction of future errors, based on current rate of change. The Sum of three actions is used to regulate the process through a control element such as the position of a control valve, a damper or the power supplied to a heating element.

The controller trusts only on the measured process variable, not on knowledge of the underlying process, making it a broadly useful controller. For specific purpose, the PID controller tuned by three parameters. The error is exist when the output overshoots set point. The use of the PID Algorithm for control does not assurance optimal control of the system to system stability. Certain applications may need using only one or two actions to provide the appropriate system control. By setting the other parameters to zero, the above action can be achieved. When there is absence of control action, PID controller called a PI, PD, P or I controller. PI controllers are common, since derivative action is sensitive to measurement noise, Due to absence of the integral it cannot be reach the attainable value.

PID controllers is used for control of various system stretched from industrial process to aircraft and ship dynamics. A linear fixed-gain PID controller are often adequate for controlling a nominal physical processed, the requirements for highperformance control with changes in operating conditions or environmental parameters are often beyond the capabilities of simple PID controllers, many approaches have been developed to improve the adaptability and robustness by adopting the selftuning methods, general predictive control, fuzzy logic and neural network strategy, and other methods.

The PID controller is the common form of feedback. PID control is an important ingredient of a distributed control systems. PID controllers are also embedded in many special purpose control systems. PID control is often combined with logic, sequential functions, selectors and simple function blocks to build the complicated automation system used for energy production, transportation and manufacturing. Many sophicated control strategies, such as model predictive control, are also organized hierarchically. For lowest level the PID control is used.

\section{B. Robust Pid Controller Tuning}

In uncertain environment the PID controller tuning is maintain the entire system as stability. Instead PID tuning and loop optimization is used to ensure consistent result. These software packages will gather the data, develop process model, and suggests optimal tuning. Some software packages can develop the tuning by gathering data from reference changes. Mathematical PID loop tuning induces an impulse in the system, and then uses the controlled systems frequency response to design the PID loop values. In loop with response times of several minutes, mathematical loop tuning is recommended, because trial and error can take days just to find a stable set-of-loop values. Optimal values are harder to find. Somme digital loop controllers offer a self-tuning feature in which very small set point changes are sent to the process, allowing the controller itself to calculate optimal tuning values. Other formulas are existing to tune the loop according to different performance criteria. Many formulas are now embedded within PID tuning software and hardware modules. Advances in automated PID loop tuning software also deliver algorithms for tuning PID loops in a dynamic or non-steady state scenario. The software will model the dynamic of a process, through a disturbance, and calculate PID control parameters in response.

\section{Proposed System}

\section{A. Energy Management Control}

The block diagram is consisting of pv array, wind turbine and battery storage devices and decentralized controller. It also connected variable loads. In figure the microgrid structure shown The microgrids haves DC to AC bus. The sources of microgrids are connected in DC bus system and load is connected to boost converter for step up for small variation of irradiation and maintain the constant voltage as $600 \mathrm{~V}$. The PMSG output voltage AC. It also affects the wind variation so it is rectifying using rectifier and it maintain $600 \mathrm{~V}$ DC. In battery storage system have the two controlled switch for energy storage and discharge. In storage switch is connected to MG. AC voltage is converted in to DC using rectifier circuit and it stored the SOC is less than $20 \%$, once it comes in charging mode the SOC goes more than $70 \%$ it is stable in charging mode or the present of DG generation is more than load. If the SOC is above $70 \%$ and load is more than DG generation the battery is connected to discharge mode.

The conversion of $\mathrm{DC}$ to $\mathrm{AC}$ is made by the voltage source inverter using voltage source controller. The VSC controller is controls the inverter thyristors, based on the MG supply frequency. The pulse generated from PWM and signal is gives VSI if The MG voltage when reaches to zero. So the grid synchronization is very easy and phase sequence is also alien. The load is variable character, it changes zero to maximum and it also three phase load. The microgrids are connected to MG using three phase circuit breakers are operated decentralized controller. The battery operation is based on SOC battery level. The decentralized controller is operation is based on the figure flow chart. The controller focus is only to satisfy the load demand for internal source or DG generated power. If the DG not satisfy the demand then battery is connected to discharge mode and again the demand is high the MG is also connected.

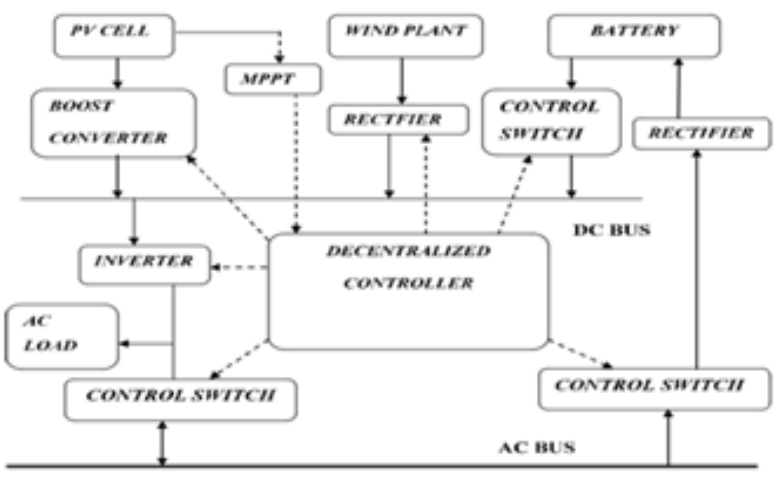

Fig. 1: Block Diagram of Proposed System

\section{B. Mode of operation}

The microgrid is having the self healing technology using decentralized controller. The controller gets all parameters of microgrid likes load power, irradiation, wind speed, battery SOC. The controller is designed FA and all parameters given as input, the algorithm is executed it operates the breakers then the microgrid operation is optimized. The microgrid basically operates in two modes

1. MG connected mode

2. Islanded mode

In MG connected mode, decentralized controller is first check the source availability of microgrid and it decides the operation. If the microgrid not satisfies the load demand and the battery SCO is less than 20\%then it takes the power from MG and the load is negligible it supply to MG at same time SOC is more than $70 \%$. The microgrid is operated in following two modes.

\section{i. $\quad$ Supply to the MG}

ii. Supply from the MG

In island mode the decentralized controller check the load; power generation of microgrid and SCO of battery. The SCO of battery is more than $20 \%$ and load demand is satisfy by microgrid power generation then the microgrid operates in islanded mode.

The aim of the microgrid is to satisfy the local demand without any disturbances and the generated power is tracks it faster. Based on the controller commend the switches are operated and the DG 
generated power is utilized and also send to MG. due to power send to MG the conventional power generation is minimized and source are saved. So the pollution also reduced.

The microgrid is connected in MG two ways. If generation of the microgrid power is exists load demand then it supply to MG at a same time the load demand is more than generation, then existed power is takes from MG if SOC is less than $20 \%$ the battery also charge and load also satisfy in MG.

The microgrid power generation is more than the load and also the battery SOC is more than the $20 \%$ the microgrid is connected to the MG and the power flow is microgrid to MG.

The microgrid power generation is fully showdown or not satisfies the load demand at the same time the battery SOC is also less than the $20 \%$ the microgrid full load condition the power flow is takes from MG to Microgrid and battery is charged. It applicable both maximum load and minimum load but SOC is less than $20 \%$.

The decentralized controller check the load, if the load is minimum level and SOC of battery condition the island mode is operated. It operates only the minimum load condition only. The battery charging and discharging is depends on SOC of battery.

The battery charging and discharging conditions are based on SOC of battery. The battery generally charge in MG only. In minimum load demand that time the microgrid tries to operate on islanded mode, so that time in discharge the energy. It is not enough to satisfy the demand that time microgrid connect to MG but the battery is operates in discharging condition.

The SOC of battery goes below the $20 \%$ that time it takes energy from the MG and it goes to charging mode. The SOC of battery goes up to $70 \%$ it is in charging mode only. Here the battery is laso act as source. The proper charging and discharging of the battery is very important. Then only the battery life will be increased.

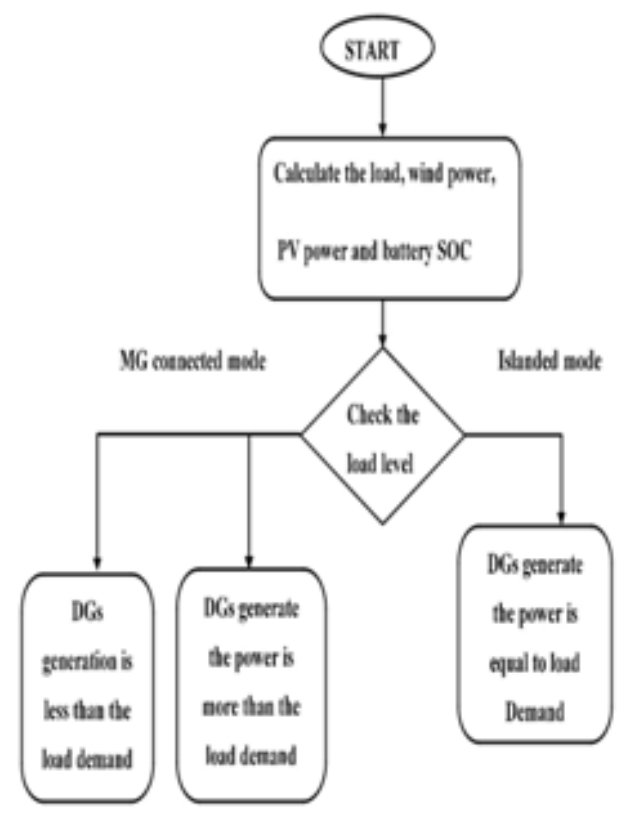

Fig. 2: Flow Diagram for Microgrid Operation

\section{Simulation Circuit and Result}

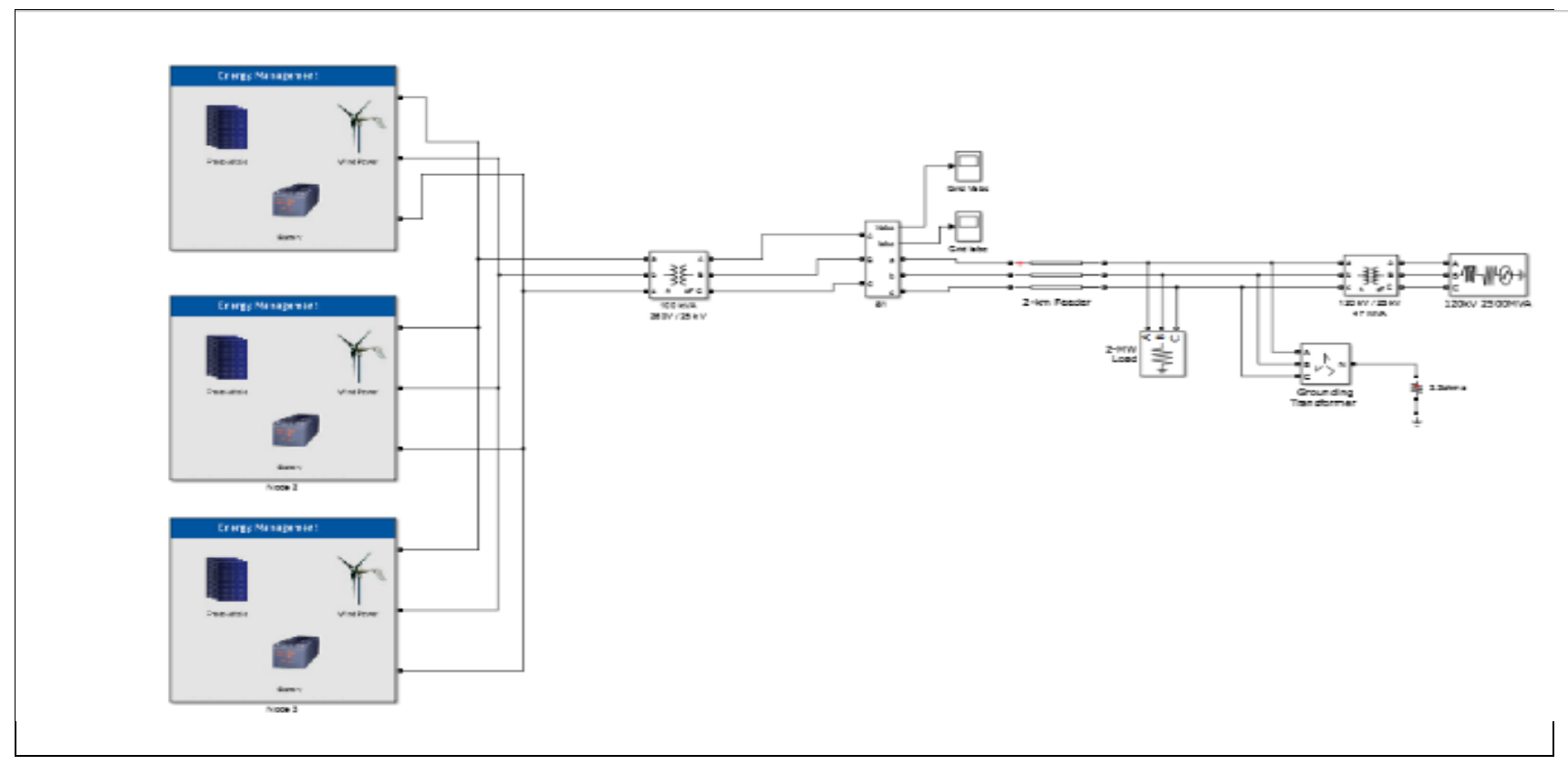

Fig. 3: Overall simulation Diagram

The simulation model was developed in Matlab/Simulink. Three mirogrid system model is shown in figure (c) the MG generator is $2500 \mathrm{KW}$ and it had the various type of loads and $2 \mathrm{~km}$ feeders line. It also $120 \mathrm{kV}$ to $25 \mathrm{kV}$ and $25 \mathrm{kV}$ to $440 \mathrm{kV}$ transformers. The three microgrids are having same amount of PV cell, wind plant, battery and loads. The microgrid has $100 \mathrm{~kW}$ PV cell, wind plant,
6.5Ah battery and $120 \mathrm{~kW}$ linear load. The PV cell output boosted $600 \mathrm{~V}$ by using boost converter. The wind generator it design at $30 \mathrm{kw} 440 \mathrm{v}$, the AC output of PMSG is rectified in to DC. The microgrid operates in minimum load less than $10 \mathrm{~kW}$. It operate islanded mode, that time the DGs and battery storage is supplies the power. 


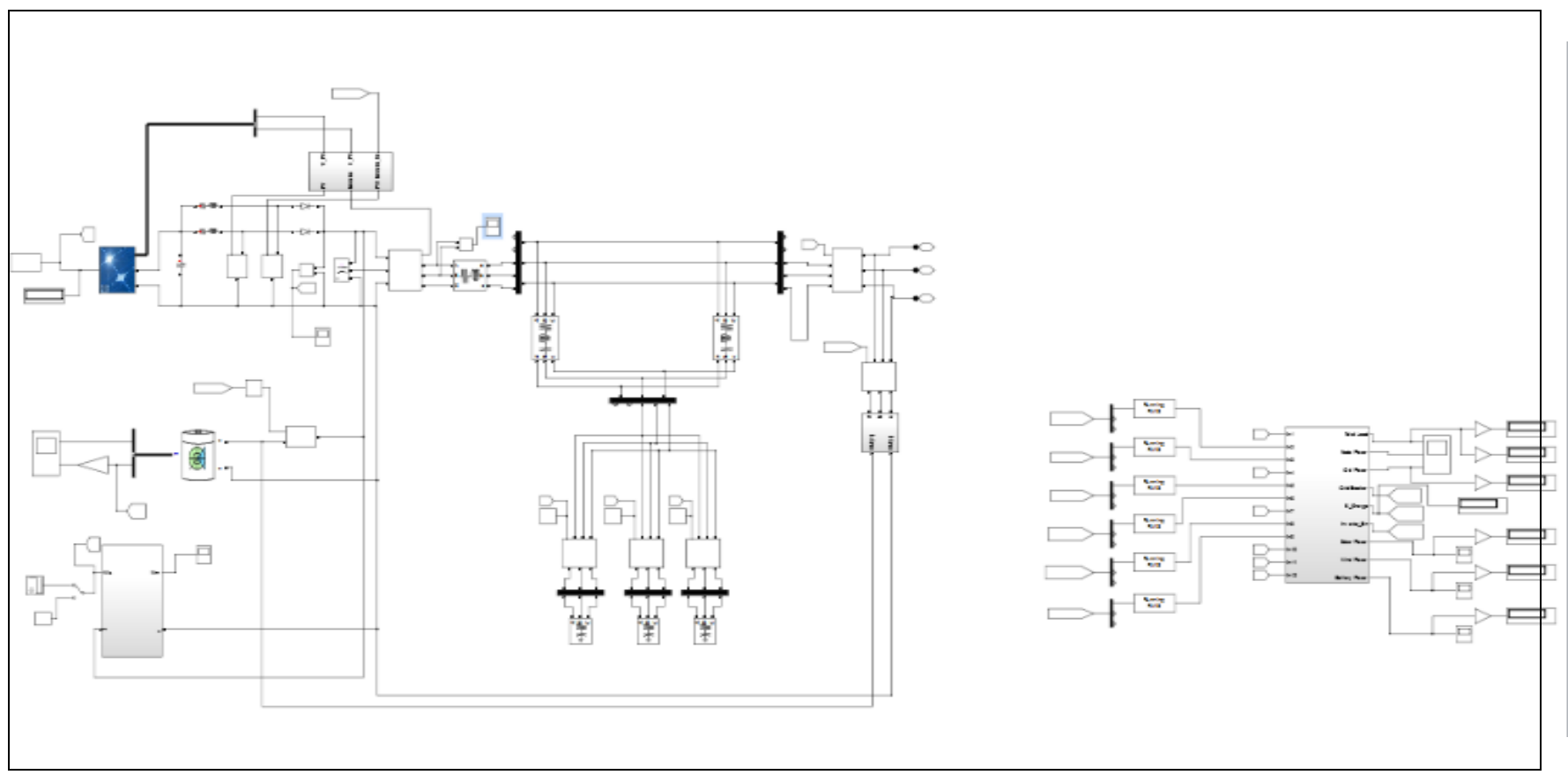

Fig. 4: Single Microgrid Diagram

The microgrid consist of DGs like PV cell, wind turbine, battery for storing the storage of power, load. Power electronics devices and control switches. The Microgrid self-healing process is controlled using decentralized controller. Figure (d) shows simulation model of Microgrid. The controller having algorithm for Microgrid. Robust optimization for self-healing and optimum usage of DG power generation. The output of PV $80 \mathrm{KW}$, wind plant $24 \mathrm{KW}$ and $100 \mathrm{Ah}$ battery. In testing purpose $120 \mathrm{KW}$ load is connected, the SCO of battery is varying. The operation is controlled by decentralized controller to operate the controlled switches.

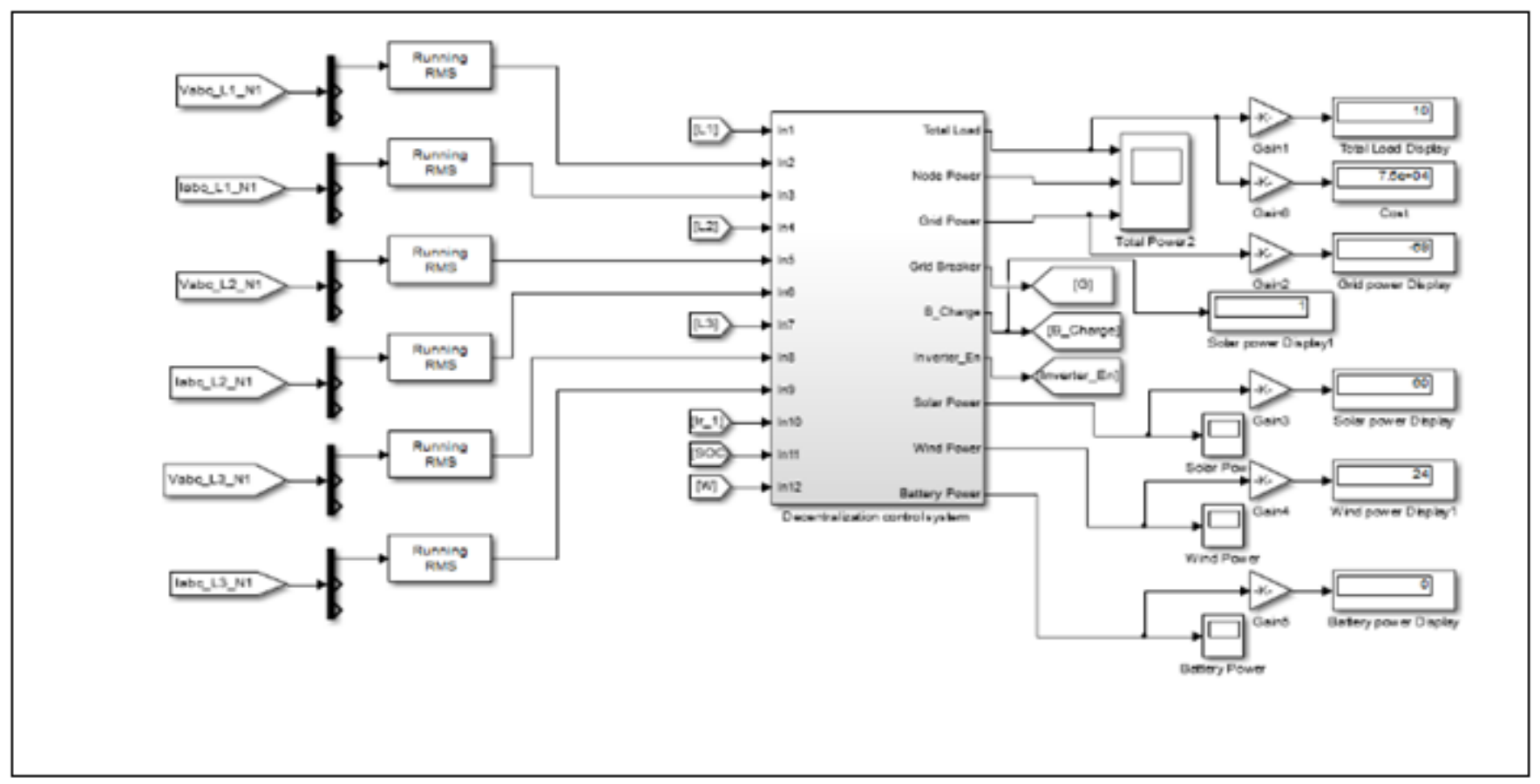

Fig. 5: Decentralized controller

The decentralized controller is operates the maximum load demand and the source power which compensate the load. The mode of operation is decided the controlling of switches. In each microgrid having the three controlled switches. The main switch is connected in three phase line, the control signal of the breaker is varied in controller connection.
The controller inputs are load voltage, load current, irradiation in sun, wind speed and battery SCO. By the various parameters the various mode of operation and the system stabilization time also discussed. The MG is connected 2500MW conventional generator, the main generator is operated the microgrid reflections. The below figure shows the voltage and current waveform. 


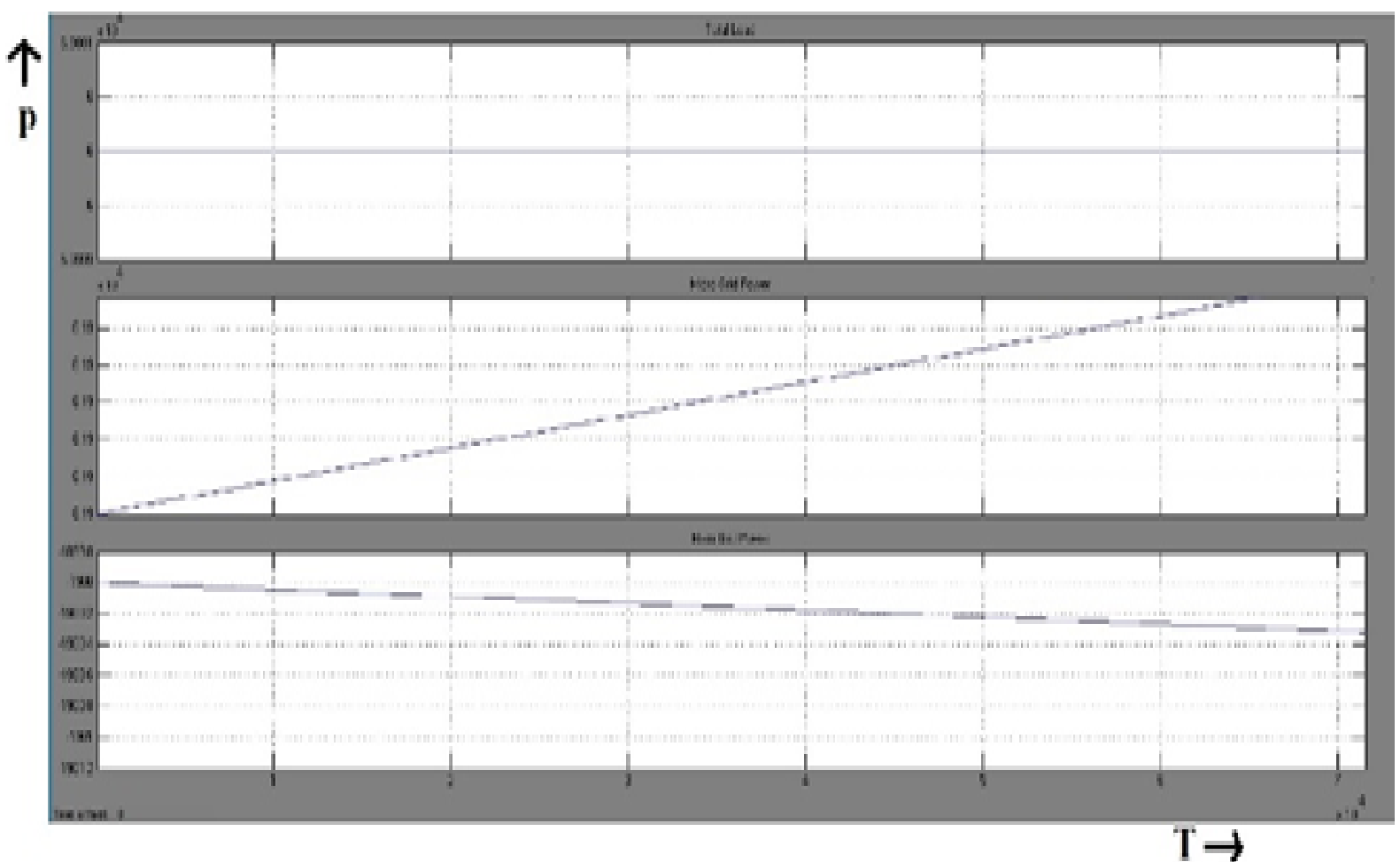

Fig. 6: Power Sharing Graph

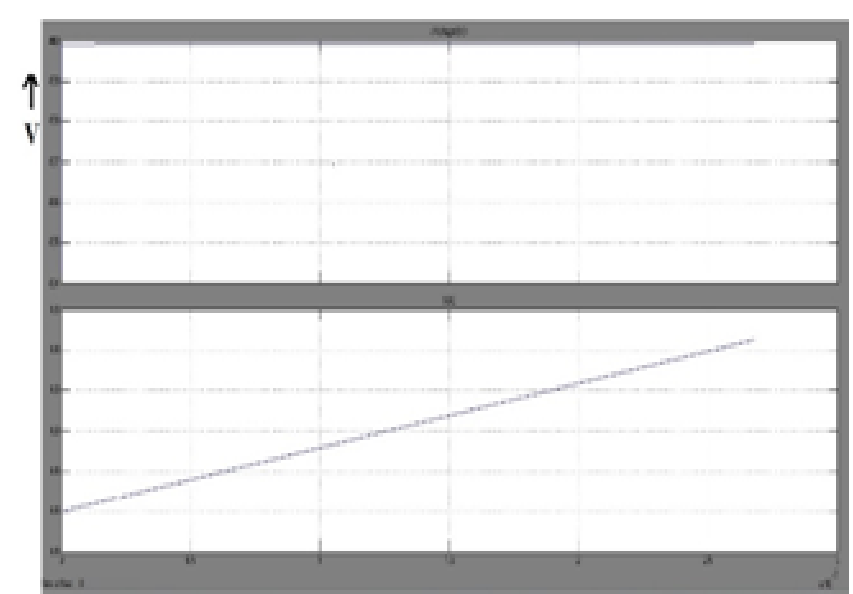

$\mathrm{T} \rightarrow$

Fig. 7: Battery SOC and Voltage

Fig 7.shows the battery charging and discharging operation. The total load of $120 \mathrm{KW}$ was satisfied DGs, Battery and MG.

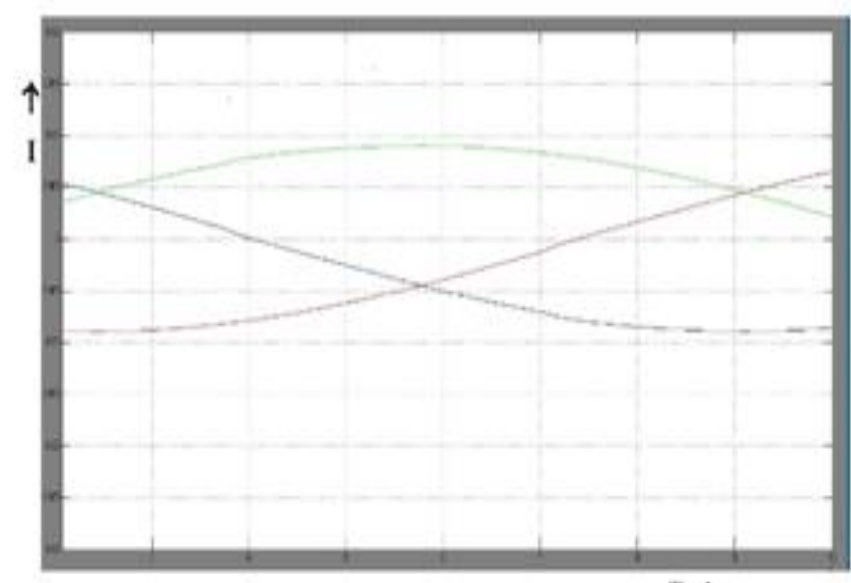

$\mathrm{T} \rightarrow$

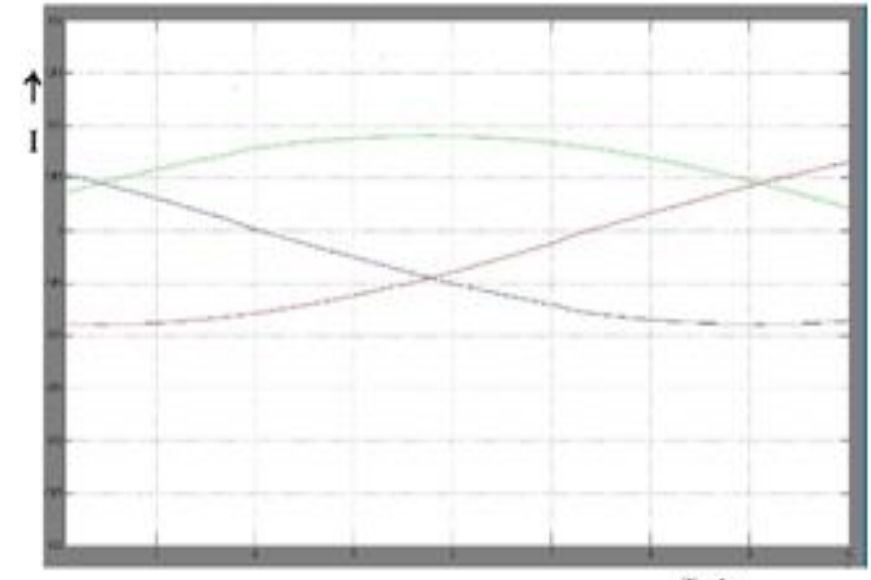

Fig. 9: Grid current

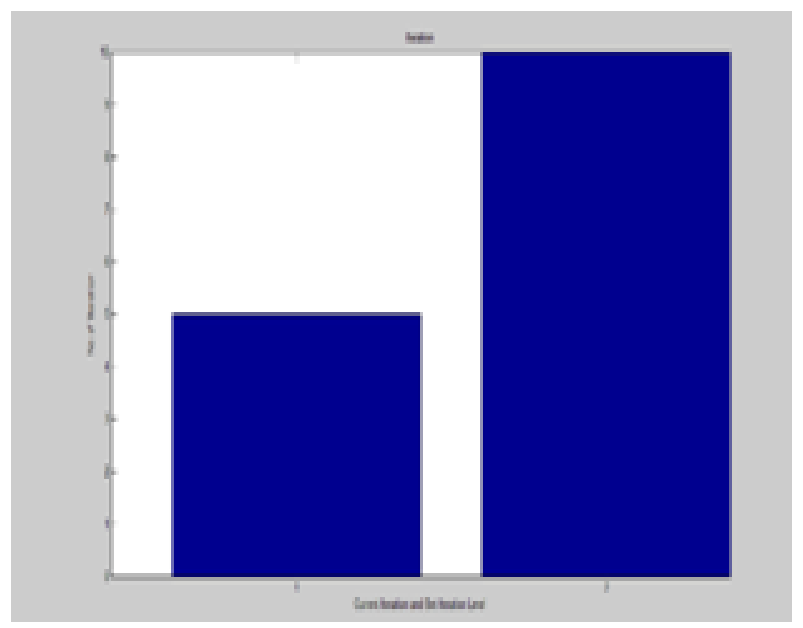

Fig. 10: Number of iteration

Fig. 8: Grid voltage 


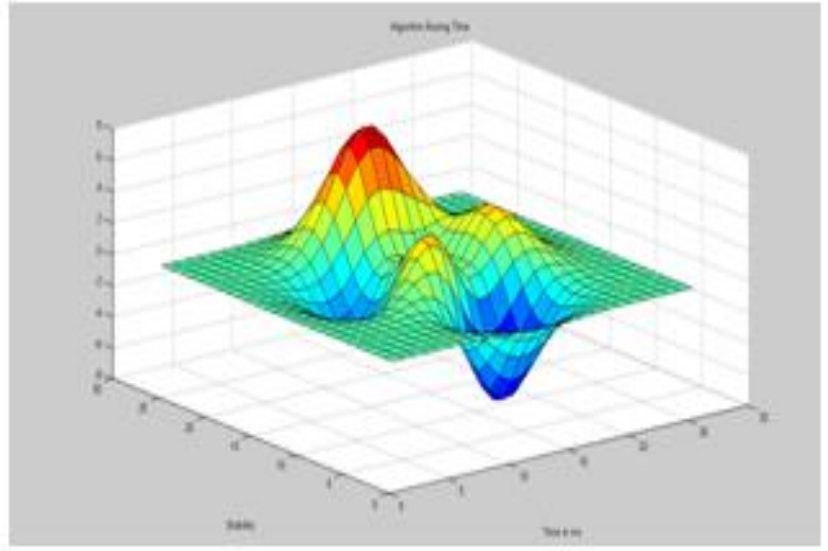

Fig 11. Computation time

Table 1. For Microgrid 1 parameters

\begin{tabular}{|c|c|c|c|c|c|}
\hline $\begin{array}{c}\text { Total } \\
\text { load } \\
(\mathbf{K W})\end{array}$ & $\begin{array}{c}\text { Grid } \\
\text { power } \\
(\mathbf{K W})\end{array}$ & $\begin{array}{c}\text { Solar } \\
\text { power } \\
(\mathbf{K W})\end{array}$ & $\begin{array}{c}\text { Solar } \\
\text { irradiation } \\
\mathbf{W} / \mathbf{m}^{\wedge} \mathbf{2}\end{array}$ & $\begin{array}{c}\text { Wind } \\
\text { power } \\
(\mathbf{K W})\end{array}$ & $\begin{array}{c}\text { Battery } \\
\text { power } \\
(\mathbf{K W})\end{array}$ \\
\hline $\mathbf{1 0}$ & -2.1 & 30 & 500 & 06 & 0.00 \\
\hline $\mathbf{1 0}$ & -79 & 30 & 500 & 24 & 35.0 \\
\hline $\mathbf{1 0}$ & -69.5 & 18 & 300 & 24 & 37.5 \\
\hline $\mathbf{1 0}$ & -81.9 & 36 & 600 & 06 & 49.9 \\
\hline $\mathbf{1 0}$ & -87.99 & 24 & 400 & 24 & 49 \\
\hline
\end{tabular}

Table 2. For Microgrid 2 parameters

\begin{tabular}{|c|c|c|c|c|c|}
\hline $\begin{array}{c}\text { Total } \\
\text { load } \\
(\mathbf{K W})\end{array}$ & $\begin{array}{c}\text { Grid } \\
\text { power } \\
(\mathbf{K W})\end{array}$ & $\begin{array}{c}\text { Solar } \\
\text { power } \\
(\mathbf{K W})\end{array}$ & $\begin{array}{c}\text { Solar } \\
\text { irradiation } \\
\mathbf{W} / \mathbf{m}^{\wedge} \mathbf{2}\end{array}$ & $\begin{array}{c}\text { Wind } \\
\text { power } \\
(\mathbf{K W})\end{array}$ & $\begin{array}{c}\text { Battery } \\
\text { power } \\
(\mathbf{K W})\end{array}$ \\
\hline $\mathbf{1 0 0}$ & 10 & 30 & 500 & 06 & 00 \\
\hline $\mathbf{1 0 0}$ & -79 & 30 & 500 & 24 & 35 \\
\hline $\mathbf{1 0 0}$ & 5 & 36 & 600 & 24 & 35 \\
\hline $\mathbf{1 0 0}$ & 57 & 42 & 700 & 06 & 00 \\
\hline $\mathbf{1 0 0}$ & -10 & 36 & 600 & 24 & 50 \\
\hline
\end{tabular}

Table 3. For Microgrid 3 parameters

\begin{tabular}{|c|c|c|c|c|c|}
\hline $\begin{array}{c}\text { Total } \\
\text { load } \\
(\mathbf{K W})\end{array}$ & $\begin{array}{c}\text { Grid } \\
\text { power } \\
(\mathbf{K W})\end{array}$ & $\begin{array}{c}\text { Solar } \\
\text { power } \\
(\mathbf{K W})\end{array}$ & $\begin{array}{c}\text { Solar } \\
\text { irradiation } \\
\text { W/m^2 }\end{array}$ & $\begin{array}{c}\text { Wind } \\
\text { power } \\
(\mathbf{K W})\end{array}$ & $\begin{array}{c}\text { Battery } \\
\text { power } \\
(\mathbf{K W})\end{array}$ \\
\hline $\mathbf{6 0}$ & -1.916 & 15.92 & 265 & 06 & 40 \\
\hline $\mathbf{6 0}$ & -29.9 & 15.9 & 265.3 & 24 & 50 \\
\hline $\mathbf{6 0}$ & -12.29 & 16.29 & 265 & 06 & 50 \\
\hline $\mathbf{6 0}$ & -1.9 & 15.9 & 265 & 06 & 40 \\
\hline $\mathbf{6 0}$ & -19 & 15.9 & 265 & 24 & 25 \\
\hline
\end{tabular}

\section{Conclusion}

A Microgrid are bidirectional power flow system. In this project small scale range of RES and loads are used for simulation. In microgrid the RES are the main source for power generation and battery is supporting source. Before the simulation running time, the parameters like sun irradiation, wind speed and battery SOC are fixed but are different in all microgrids. During simulation running time load values are possible to change manually. If any changes in the microgrids, the demand is compensate by DG or MG or combined both.

The microgrids are connected with MG. The source of microgrids are $100 \mathrm{KW}$ of PV cell, $30 \mathrm{KW}$ of PMSG wind plant and $6.5 \mathrm{Ah}$ battery. The load range of each microgrid is $120 \mathrm{KW}$ linear load. The decentralized controller is used for self-healing process, because the parameters are dynamically or manually changed. The operation is based on load voltage, current, sun irradiation, wind speed, battery SCO and then the controller decides the operation of microgrid is islanded mode or MG connected mode. If the load demand is equal to DG power generation the microgrid is operate in islanded mode. Otherwise the load demand is exceeds the generation or lower than the generation the microgrid are operated in MG connected mode.

During the changes in microgrids, to satisfy the demand with minimum tracking time and to stabilize the system, proper algorithm is needed to control the switches and operate the converter and inverter. The computation time is different for various algorithms. In this robust optimization contour plot, number of iteration are shown. In robust optimization the number of iteration is 4 and computation time is 40 to $50 \mathrm{~ms}$.

\section{References}

[1] Ali Mehrizi-sani, graduate and rezairavani, (2010), "PotentialFunction Based Control of a Microgrid in Islanded and GridConnected Modes", IEEE transcations on power systems, Vol. 25, no. 4, pp. 324-340.

[2] Alirezakahrobaeian, Yasser Abdel and Radyk I. Mohamad,(2012), "Distributed Generation Interface for Flexible Micro-Grid Operation In Msrt Distribution System", IEEE Transaction On Sustainable Energy, vol. 3, no. 2, pp. 295-305.

[3] Daniel E. Olivares, Ali Mehrizi-Sani, (2014), "Trends in Microgrid Control,", IEEE transactions on smart grid vol. 5 no. 4 pp. 1905-1919.

[4] Chao Lin, Josep M. Guerrero, XiaochaoHou ,XiaofengWang, Yao Liu, (2013) "Multi-Agesnt Based Hierarchical Hybrid Control For Smart Microgrid", IEEE transactions on smart gridvol 4, no. 2, pp. 771-778.

[5] Mehmet H. Cintuglu, and Osama A. Mohammed(2015), "Multiagent-Based Decentralized Operation of Microgrids Considering Data Interoperability"Smart Grid Communications, IEEE International Conference.

[6] Shadmand, M.B.; Balog, R.S.,(2014) "Multi-Objective Optimization and Design of Photovoltaic-Wind Hybrid System for Community Smart DC Microgrid", in Smart Grid, IEEE Transactions on, vol.5, no.5, pp.2635- 2643.

[7] YinliangXu; Zhicheng Li(2015), "Distributed Optimal Resource Management Based on the Consensus Algorithm in a Microgrid", in Industrial Electronics, IEEE Transactions on, vol.62, no.4, pp.2584-2592.

[8] TossapornSurinkaew and IssarachaiNgamroo(2014), "Robus Power Oscillation Damper Design For DFIG-Based Wind Turbine Based On Specified Structure Mixed H2/HN Control", ELSEVER transactions on renewable energy, vol 66,PP. 15-24.

[9] Jianze Wang, Ningning Li, YanchaoJi ,Yiqi Liu, Yu Fu, (2015)"Enhanced Load Power Sharing Accuracy in DroopControlled DC Microgrids with Both Mesh and Radial Configurations", Energies, ISSN 1996-1073.

[10] F. Y. S. Eddy and H. B. Gooi(2011), "Multi-Agent System For Optimization of Microgrids", Power Electronics and ECCE Asia (ICPE \& ECCE), IEEE 8th International Conference, pp. 23742381

[11] PawelMalysz, ShahinSirouspour, AliEmad (2015) "“An Optimal Energy Storage Control Strategy for Grid-connected Microgrids" Smart Grid Communications IEEE International Conference.

[12] Jiang Wu, Xiaohong Guan,(2013) "Coordinated MultiMicrogrids Optimal Control Algorithm for Smart Distribution Management System" IEEE Transcations on Smart Grid vol. 4 no.4 pp. $2174-2181$

[13] Quanyuan Jiang, eidongXue, and GuangchaoGeng (2013), "Energy Management of Microgrid in Grid-Connected and Stand-Alone Modes", vol. 28, no.3, pp.3380 - 3389

[14] Tine L. Vandoorn, jeroen de kooning (2013) "microgrids Hierarchical Control and an Overview of the Control and Reserve Management Strategies", IEEE Industrial Electronics Magazine, vol. 7, no. 4, pp. 42-55

[15] Alessandra Parisio, EvangelosRikos, and Luigi Glielmo (2014), "A Model Predictive Control Approach to Microgrid Operation Optimization", IEEE Transactions on Control Systems Technology, vol. 22, no. 5, pp. 1813 - 1827.

[16] Stefano Bracco, Massimo Brignone, Federico Delfino (2015), "An Energy Management System for the Savona Campus Smart Polygeneration Microgrid", IEEE Systems Journal, vol 11 , no. 3, pp. 1799 - 1809 
[17] SeyedaliMoayedi, Ali Davoudi (2016), "Unifying Distributed Dynamic Optimization and Control of Islanded DC Microgrids", Journal of Energy Engineering, American Society of Civil Engineers vol. 32,no. 3 pp. 2329 - 2346.

[18] T. Padmapriya and V.Saminadan, "Improving Performance of Downlink LTE-Advanced Networks Using Advanced Networks Using Advanced feedback Mechanisms and SINR Model", International Conference on Emerging Technology (ICET), vol.7, no.1, pp: 93, March 2014.

[19] S.V.Manikanthan and D.Sugandhi “ Interference Alignment Techniques For Mimo Multicell Based On Relay Interference Broadcast Channel " International Journal of Emerging Technology in Computer Science \& Electronics (IJETCSE) ISSN: 0976-1353 Volume- 7 ,Issue 1 -MARCH 2014. 